\section{HEPATITIS B}

\section{Informationsaktion für Migranten}

Nach dem Auftakt der Informationsaktion von Novartis über chronische Hepatitis B „Aufklärung schützt die Leber!“ mit einer mehrsprachigen Infobroschüre im Jahr 2008, wurde diese nun aufgrund der guten Resonanz zielgruppenspezifisch aufbereitet, erweitert und neu herausgegeben. Wichtige Informationen rund um die Gefahren, Symptome und Behandlungsmöglichkeiten einer der nach wie vor häufigsten Infektionskrankheiten sind auf Deutsch, Englisch, Bosnisch-KroatischSerbisch, Türkisch, Rumänisch und Russisch enthalten und richten sich verstärkt an in Österreich lebende Migranten.

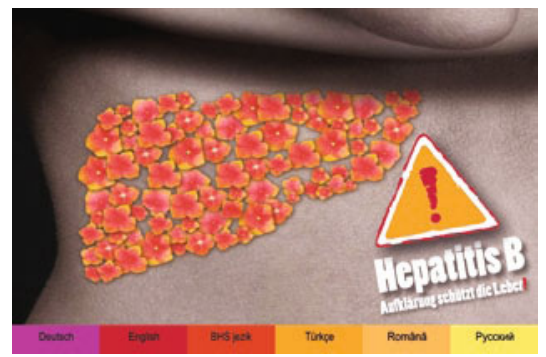

Mehrsprachige Broschüre über Hepatitis B für bessere Information von Migranten

WHO-Schätzungen zufolge leben in Österreich etwa 42.000 chronische Virusträger, jährlich werden bis zu 5.000 Personen neu infiziert. In Österreich mehr als 1,4 Mio. Personen mit Migrationshintergrund (17,5 Prozent der Gesamtbevölkerung). Eine Förderung der Chancengleichheit und ein erleichterter Zugang zu Informationen für alle in Österreich lebenden Personen beim Erhalt von Gesundheitsdienstleistungen ist deshalb ein wichtiges gesamtgesellschaftliches Ziel, aber der Zugang zu präventiven und gesundheitsfördernden Angeboten im Gesundheitsbereich ist für Migranten oft erschwert. Das Fehlen von zugänglichen Informationen in ihrer Muttersprache sowie sprachlich-kulturell und fachlich kompetenten Ansprechpersonen, bilden vor allem aus Sicht von älteren Migranten zwei wesentliche Barrieren für die Inanspruchnahme von gesundheitsfördernden Angeboten.

Die neu überarbeitete Informationsbroschüre über chronische Hepatitis $B$ und die in diesem Zusammenhang angeführten Kontakte zu Ärzten, Gesundheitseinrichtungen und migrantischen Vereinen sollen einen Beitrag dazu leisten, die
Kommunikationsbarrieren zwischen den verschiedenen Sprachgruppen zu lösen und Gesundheitsrisiken aufgrund von mangelndem Verständnis zu vermeiden.

Für Fachärzte und Ordinationen gibt es die Möglichkeit, die neue Hepatitis BInfobroschüre unter der Faxnummer 01/319 144440 kostenlos zu bestellen.

Informationen: Mag. Barbara Frueh-Senkyr, Novartis Pharma GmbH, Tel.: 01/866 57-6360, E-Mail: barbara.frueh-senkyr@novartis.com, Internet: www.hepatitis-info.at

\section{ARABMED IN EUROPE}

\section{Jahrestreffen diesmal in Irland}

Um den zahlreichen in Europa tätigen Ärzte aus dem arabischen Kulturraum eine Plattform zu geben, wurde 1983 in Deutschland die Union Arabischer Mediziner in Europa (ARABMED) gegründet. Aufgaben sind wissenschaftliche, soziale und humanitäre Maßnahmen sowie die Vertiefung freundschaftlicher Beziehungen zwischen den Kollegen und deren Familien. Die Union möchte eine Brücke zwischen den Ärzten in Europa und der arabischen Welt schlagen, um einen Beitrag in der Weitervermittlung der aktuellen Entwicklungen der medizinschen Welt zu leisten.

Zu den Aktivitäten zählen die Herausgabe einer Medizinischen Zeitschrift in arabischer Sprache sowie die Organisation von jährlichen Kongressen und sonstigen Veranstaltungen mit Betonung der Berufsethik. Der diesjährige Kongress findet in

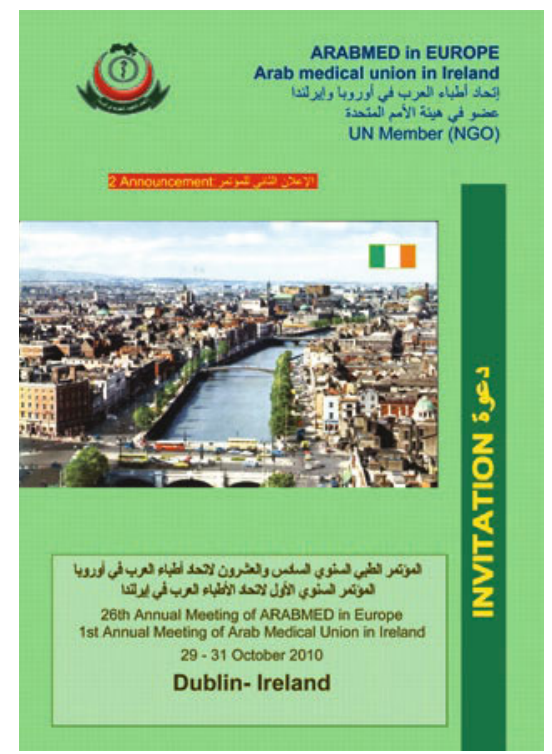

Plattform für Erfahrungsaustausch und ethisches Handeln: ARABMED in Europe
Dublin/Irland vom 29. bis 31. Oktober statt. An fachlichen Themen stehen Herzerkrankungen, Stoffwechselkrankheiten, Augenheilkunde, allgemeine, laparoskopische und Unfallchirurgie, Urologie, orthopädische und Plastische Chirurgie, Adipositas im Kindesalter, Anästhesie und Intensivmedizin, Verbrennungsverletzungen, Gynäkologie und Geburtshilfe und medizinische und pharmazeutische Ethik auf dem Programm.

\section{ARABMED in EUROPE}

Termin: 29. bis 31. Oktober 2010

Informationen: Dr.Ghassan Elagha, Präsident der Arabischen medizinischen Union in Irland 2 Temple Manor Grove, Limekil avenue, Dublin 12, Irland, Tel.: 0035/3868038162, E-Mail:ghassanalagha@hotmail.com

\section{MARKETING}

\section{Goldenes Skalpell für Multaq ${ }^{\circledR}$ Tablet-PC E-Detail}

Mit dem Goldenen Skalpell im Rahmen des „Best of Pharma Advertising“ wurde heuer das Projekt Multaq ${ }^{\circledR}$ Tablet-PC von sanofiaventis und der Agentur swot in der Kategorie E-Marketing ausgezeichnet. Mit dem neuen Kommunikationskanal Tablet-PC EDetail können die Pharmaberater im Gespräch ganz auf die individuellen Kundenbedürfnisse eingehen, man kann Inhalte kompakt vermitteln, aber auch sehr leicht und rasch in ein Spezialgebiet eintauchen. Ein Tablet-PC ist ein tragbares stiftbedienbares Notebook. Der Benutzer kann dabei im Gegensatz zu herkömmlichen Notebooks Eingaben per Stift direkt auf dem Bildschirm tätigen und durch eine digitale Präsentation (auch E-Detail genannt) navigieren. Mittels E-Detailing können Ärzte mit Informationen über Multaq ${ }^{\circledR}$ in elektronischer Form versorgt werden.

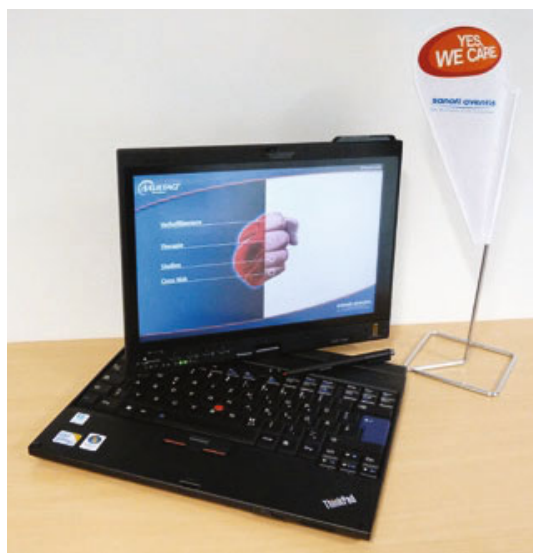

Der Tablet PC verbessert die Kommunikation zwischen Arzt und Berater. 